\title{
Avaliação de programa sobre identificação de emoções por alunos do ensino básico
}

Assessment of a program to identify expression of emotions by primary school students Evaluación del programa sobre identificación de emociones por alumnos de educación primaria

Sofia Margarida Guedes de Campos iD https://orcid.org/0000-0002-4696-3537 Maria da Graça Aparício Costa iD https://orcid.org/0000-0001-6149-062X

Manuela Ferreira iD https://orcid.org/0000-0003-4512-1669

Olivério Paiva Ribeiro iD https://orcid.org/0000-0001-7396-639X

Sandra Costa io hitps://orcid.org/0000-0001-9995-5787

João Duarte ic https://orcid.org/0000-0002-2390-3910

Rosa Martins id https://orcid.org/0000-0003-1842-4840

Carlos Albuquerque io https://orcid.org/0000-0002-2297-0636 ${ }^{1}$

\section{Como citar:}

Campos SM, Costa MG, Ferreira M, Ribeiro

OP, Costa S, Duarte J, et al. Avaliação de programa sobre identificação de emoções por alunos do ensino básico. Acta Paul Enferm. 2020;33:e-APE20190049

DOI

http://dx.doi.org/10.37689/ acta-ape/2020A00049

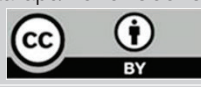

De; Instituições acadêmicas; Educação em saúde

Keywords

Emotions; Child; Adolescent; Schools; Health education

\section{Descriptores}

Emociones; Niño; Adolescente; Instituciones académicas; Educación en Salud

Submetido 8 de Março de 2019

Aceito

22 de Julho de 2019

\section{Autor correspondente}

Sofia Campos

Rua Dom João Crisóstomo Gomes de Almeida, 102, 3500-843, Viseu, Portugal.

http://orcid.org/0000-0002-4696-3537

E-mail: sofiamargaridacampos@gmail.com

\section{Resumo}

Objetivo: Avaliar a eficácia de um programa de educação sócio emocional na diferenciação e identificação de emoções em alunos do $2^{\circ}$ e $3^{\circ}$ ciclos do Ensino Básico e analisar a influência das variáveis sociodemográficas.

Métodos: Estudo de intervenção com avaliação antes e depois, realizado numa amostra de 101 alunos, 58,4\% raparigas, divididos nos grupos etários de 9-11 (média= 10,34 anos; $D p=0,59$ ) e 12-15 anos (Média= 13,37; $\mathrm{D}=$ = 0,99). Foi aplicado o Inventário de Identificação de Emoções e Sentimentos (IIES- Moreira, P., Oliveira, J.T., Crusellas, L., Lima, A., 2012) antes e após programa de educação socio emocional, baseado no Programa "expressões faciais de 6 emoções básicas dos Mochinhos da Sabedoria" de Costa (2012).

Resultados: Os alunos tinham entre 9 e 15 anos e a maioria $(27,7 \%)$ frequentava $08^{\circ}$ ano de escolaridade. A capacidade de identificar e diferenciar emoções e sentimentos variou significativamente em função do género e idade. A comparação de médias na amostra global não revelou significância estatística na diferenciação e identificação de emoções antes e após a intervenção. Analisando por grupos etários o Test-t para diferença de médias indicou diferenças significativas na capacidade de identificação emocional positiva $(t=-2,813 ; p=$ $0,007)$ e diminuição na identificação emocional neutra $(t=2,258 ; p=0,029)$ no grupo dos 9-11 anos.

Conclusão: 0s resultados encontrados indicam que as competências de diferenciação e identificação de emoções se alteram após estratégias educacionais, sobretudo em idades precoces. Isto terá efeito na autoregulação emocional e nas experiências emocionais quotidianas com os outros, sobretudo se incluídas de forma intencional e sistemática em ambiente escolar.

\section{Abstract}

Objective: to assess the efficacy of a socioemotional education program on recognizing and distinguishing emotions among 2nd and 3rd cycle primary education students and analyze the influence of sociodemographic variables.

Methods: This was a before-after intervention study including a sample of 101 students (58.4\% girls) divided into two groups. The first groups age ranged from 9 to 11 years-old (mean: 10.34 years; SD $=0.59$ ) and in the second group from 12 to 15 years-old (mean: $13.37 ; \mathrm{SD}=0.99$ ). We applied the Emotions and Feelings Identification Awareness Inventory (IIES- Moreira, P., Oliveira, J.T., Crusellas, L., Lima, A., 2012) before and after socioemotional education program based on Costa's (2012) program "6 basic emotions and facial expression by the little wisps of wisdom".

Results: Students were aged between 9 and 15 years, and most of them (27.7\%) were attending the 8th school year. The ability of recognizing and distinguishing emotions and feelings varied significantly by sex and age. The comparison of means in the global sample was not statistically significance in the identification and 
differentiation of feelings before and after the intervention. Considering students by age group, the Student t-test for mean differences showed significant differences in the ability of identifying positive emotions ( $t=-2.813 ; p=0.007)$ and a decrease in identifying neutral emotions $(t=2,258 ; p=0.029)$ in the 9-11 years-old group.

Conclusion: Findings indicate that competences in identifying and differentiating emotions change after exposition to educational strategies, especially among younger individuals. These strategies have also an effect on emotional self-regulation and on daily emotional experiences with others, mainly when they are intentional and systematically introduced into a school environment.

\section{Resumen}

Objetivo: Evaluar la eficacia de un programa de educación socioemocional para la diferenciación e identificación de emociones en alumnos de $5^{\circ}$ a $9^{\circ}$ año de primaria y analizar la influencia de las variables sociodemográficas.

Métodos: Estudio experimental con evaluación antes y después, realizado con una muestra de 101 alumnos, 58,4\% mujeres, divididos en grupos de edad de 9 a 11 (promedio= 10,34 años; $D p=0,59$ ) y 12 a 15 años (promedio=13,37; $D p=0,99$ ). Se aplicó el Inventario de Identificación de Emociones y Sentimientos (IIES- Moreira, P., Oliveira, J.T., Crusellas, L., Lima, A., 2012) antes y después del programa de educación socioemocional, basado en el programa "Expresiones faciales de seis emociones básicas" de Mocinhos da Sabedoria de Costa (2012).

Resultados: Los alumnos tenían entre 9 y 15 años y la mayoría $(27,7 \%)$ estaba en $8^{\circ}$ año de escuela. La capacidad de identificar y diferenciar emociones y sentimientos varió significativamente en función del género y la edad. La comparación de promedios de la muestra global no reveló significación estadística en la diferenciación e identificación de emociones antes y después de la intervención. Al analizar por grupos de edad, el test-T para diferencias de promedios indicó diferencias significativas en la capacidad de identificación emocional positiva $(t=-2,813 ; p=0,007)$ y reducción en la identificación emocional neutra $(t=2,258 ; p=0,029)$ en el grupo de 9 a 11 años.

Conclusión: Los resultados encontrados indican que las competencias de diferenciación e identificación de emociones se alteran luego de aplicar estrategias educativas, sobre todo en edades precoces. Esto tendrá un efecto en la autorregulación emocional y en las experiencias emocionales cotidianas con los otros, sobre todo si se incluyen de forma intencional y sistemática en el ambiente escolar.

\section{Introdução}

As emoçóes, no sentido estrito tais como a alegria, a mágoa, o medo, o orgulho, a vergonha, a simpatia, são um meio natural de avaliar o ambiente que nos rodeia e reagir de forma adaptativa. É nas emoçóes que encontramos a regulação das nossas vidas. Assim é bastante importante começar a dar valor às emoçóes e atribuir-lhes um sentido, de forma a estabelecer relaçôes mais harmoniosas, com o principal objetivo de manter o sistema emocional em equilíbrio, dada a influência protetora que as emoções exercem a nível de processos como a cognição, perceção e comportamentos. ${ }^{(1)}$

Há uma série de habilidades emocionais, cognitivas e comportamentais que se desenvolvem precocemente na vida, constituindo as relaçóes de apego estáveis com os pais/cuidadores as bases para o funcionamento socioemocional do bebé, que ajudam a promover relaçóes positivas no presente e para o futuro. $^{(2)}$ A capacidade de regular emoçóes, inibir impulsos, compreender as mensagens, ou seja, o desenvolvimento progressivo de competências emocionais tem um impacto fundamental no desenvolvimento da criança. As investigaçóes em Inteligência Emocional, ou seja, da capacidade para perceber, avaliar e expressar emoçóes ${ }^{(3)}$ têm demonstrado que as pessoas com resultados mais elevados apresentam uma melhor saúde física e psicológica, melhores relações interpessoais, mais estados emocionais positivos e, portanto, uma atitude mais positiva em relação à escola aos professores e um maior bem-estar. Assim, a aprendizagem socioemocional, enquanto processo de aquisição e aplicação eficaz de conhecimentos, atitudes e competências necessárias para reconhecer e gerir emoçóes, deve ser promovida precocemente, idealmente durante o ensino pré-primário, no sentido de desenvolver as competências básicas, tais como a autoconsciência das próprias emoções, a consciência social (empatia e respeito pelos outros), autocontrolo e competências socias (comunicação, cooperação), que por sua vez terão efeitos positivos na atenção/ concentração e desempenho escolar. ${ }^{(4)}$ Em Portugal, o Programa Nacional de Saúde Escolar (PNSE) ${ }^{(5)}$ desafia as escolas para a concretizaçáo de atividades que promovam o desenvolvimento de competências socioemocionais, dado que estas ajudam os alunos a tornarem-se mais resilientes, permite-lhes reconhecer as suas próprias emoçóes e a maneira mais adequada de lidar com elas e gerir de forma mais responsável a tomada de decisóes, sejam relacionadas com a sua saúde ou com a sua vida. A saúde escolar em Portugal promove um programa de parceria entre a Saúde Comunitária e a Educação, para a promoção da saú- 
de e prevenção da doença em contexto escolar. Estas atividades, realizadas pelas equipas das Unidades de Cuidados na Comunidade, são dirigidas às necessidades identificadas em cada Agrupamento de escolas. Com a melhoria do estado de saúde da população infantil portuguesa, o programa de saúde escolar, centra o alvo do seu paradigma na promoção de competências socio emocionais nas crianças e adolescentes e melhoria da qualidade de vida. "Melhorar a saúde e a qualidade de vida, mais do que capacitar as pessoas e as comunidades para agir, implica reconhecer as suas competências e potencialidades e facilitar as suas escolhas, grandes desafios da contemporaneidade". ${ }^{(5)}$

Neste sentido, o objetivo do nosso estudo foi avaliar a eficácia de um programa de educação socio emocional na identificação de emoçóes em alunos do ensino básico e analisar a influência das variáveis sociodemográficas.

\section{Métodos}

Estudo de intervenção com avaliação de variáveis antes e depois, desenvolvido no âmbito do Projeto MaiSaúdeMental com referência CENTRO-010145-FEDER-023293, a decorrer na Escola Superior de Saúde do Instituto Politécnico de Viseu, Portugal. Foi realizado numa amostra de conveniência constituída por 101 crianças/adolescentes 58,4\% raparigas, com idades compreendidas entre os 9 e 15 anos (mé$\mathrm{dia}=11,96$ anos; $\mathrm{Dp}=1,73)$, alunos dos $2^{\circ}$ e $3^{\circ}$ ciclos do ensino básico de um Agrupamento Escolar público da região, parceiro no Projeto MaiSaúdeMental. Os alunos encontravam-se a realizar uma visita no âmbito da Orientação Vocacional aos laboratórios da Escola Superior de Saúde de Viseu, e foram convidados a participar no estudo de intervenção aplicado por uma Psicóloga da equipa. Atendendo às diferentes competências do desenvolvimento, decorrentes da variabilidade das idades da amostra, para a análise dos dados foram considerados dois grupos etários, de 9-11 e 12-15 anos. Foi utilizado um questionário de caracterização sociodemográfica e o Inventário de Identificação de Emoçôes e Sentimentos (IIES) ${ }^{(6)}$ Este inventário de auto-relato avalia a capacidade das crianças/adolescentes identificarem e diferen- ciarem emoçóes, partindo de situaçóes quotidianas. É composto por 15 itens de resposta dicotómica e sinalização aberta das emoçôes identificadas. As respostas obtidas são posteriormente agrupadas em 4 grupos: ( 1 =Ausência de emoção/sentimento; 2 = incongruente; 3 = congruente e 4 = consensual) e distribuídos por 3 escalas: a) Valência Negativa itens associados à experiência de emoçóes de tonalidade negativa.; b) Valência Positiva - itens associados à experiência de emoçóes positivas. A soma dos itens de cada uma destas escalas permite obter o Índice de Diferenciação Emocional Negativa e Positiva respetivamente. A soma destas duas escalas permite obter o Índice de Diferenciação Emocional Total. c) Valência Neutra - itens de situações não associadas a qualquer tipo de experiência emocional. A soma das 3 escalas permite avaliar o Índice de Identificação Emocional das crianças. O programa de Educação Sócio Emocional dos Mochinhos da Sabedoria baseia-se no modelo de Inteligência Emocional de Mayer e Salovey ${ }^{(7)}$ que visa o desenvolvimento de competências da Perceção Emocional (PE), desenvolvido pelo jogo "das expressóes faciais de 6 emoçóes básicas”. O objetivo é o reconhecimento de diferentes emoçôes em si e nos outros de maneira acurada, pelo que a intervenção visava estimular a habilidade para identificar emoçôes nas outras pessoas, através da expressão facial. Segundo o autor, as leituras adequadas das expressóes e linguagem corporal do outro são fundamentais nas interações humanas. ${ }^{(8)}$ Após a aplicação do pré-teste referido anteriormente, os alunos foram sujeitos ao programa de intervenção, que consistia em apresentar-lhes a face da "Maria", à luz da emoção correspondente: o medo, a surpresa, a raiva, a tristeza, a aversão e a alegria. As crianças e jovens foram depois convidadas a experimentarem a expressão facial de cada emoção, sendo analisadas as diferenças da parte superior e inferior do rosto. Posteriormente, os alunos foram instruídos a lançar um dado de modo a ser selecionada aleatoriamente uma imagem e a nomear a respetiva emoção, bem como a partilhar situaçóes quer da escola, do grupo de pares ou da família em que essa emoção tivesse sido vivida. Também foram dados cartóes com situações nos três contextos anteriormente referidos, em que as 
crianças ou jovens tinham de identificar a emoção e a expressão facial inerente. Após a intervenção, foi aplicado o pós-teste, no sentido de se perceber se havia uma melhoria na identificação e diferenciação emocional.

A salvaguarda dos requisitos éticos foi assegurada mediante submissão prévia do protocolo do estudo à Comissão de Proteção de dados, à Direção Geral de Educação e após a sua aprovação, foi solicitada autorização ao Conselho Diretivo do Agrupamento de Escolas e obtido o consentimento informado dos Pais/Encarregados de Educação. No tratamento dos dados foi utilizado o programa informático Statistical Package for the Social Sciences ${ }^{\circledR}$ (SPSS versão 24.0) efetuando-se análise descritiva e analítica com testes paramétricos e não-paramétricos em função do tamanho da amostra, considerando-se para análise dos testes o Intervalo de Confiança (IC) a 95\% (significância $\mathrm{p}<0,05$ )

\section{Resultados}

Tal como referido, a análise dos dados foi efectuada considerando dois grupos etários, as crianças de 9-11 anos e os adolescentes de 12-15. As crianças entre 9-11 anos, no total de 47 (53,2\%) eram do sexo feminino, tinham uma média de idades de 10,34 anos $(\mathrm{Dp}=0,59)$, com valores sobreponíveis entre os sexos, e 48,9\% frequentavam o $5^{\circ}$ ano de escolaridade. No grupo etário dos 12-15 anos, num total de 54, (63,0\% do sexo feminino), a média de idades era de $13,37(\mathrm{Dp}=0,99)$, um pouco mais elevada no sexo feminino $(13,64 ; \mathrm{Dp}=0,98 ; 12,90$; $\mathrm{D} p=0,85$ respetivamente) e $51,9 \%$ frequentavam o $8^{\circ}$ ano de escolaridade, seguidos de $20,4 \%$ que frequentavam o $7^{\circ}$ ano.

Analisando as respostas obtidas relativamente ao género e face à amostra global, o teste de U-Mann Whitney dado o tamanho amostral, evidenciou ordenaçôes médias mais elevadas para o sexo feminino, quer antes quer depois do programa de educação socio-emocional. Analisando face aos grupos, verifica-se a mesma tendência face ao género feminino, apurando-se, no grupo dos 9-11 anos e antes do programa, diferenças significativas na capacida- de de identificação emocional (UMW= 157.000; $\mathrm{p}=0,011)$ e após o programa na diferenciação emocional neutra (UMW=180.5000; $\mathrm{p}=0,041)$ e identificação emocional $(U M W=171.000 ; p=0,024)$ (Tabela 1).

Tabela 1. Teste de U-Mann Whitney antes e depois do programa de intervenção no grupo de crianças, em função do sexo

\begin{tabular}{llllll}
\hline & & Masc. & Femin. & \multicolumn{2}{c}{ Teste } \\
& & \multicolumn{2}{c}{$9-11$} & UMW & $p$-value \\
\hline \multirow{2}{*}{ Antes } & Diferenciação Emocional Positiva & 23,36 & 24,56 & 261,00 & 0,705 \\
& Diferenciação Emocional Negativa & 22,25 & 25,54 & 236,50 & 0,365 \\
& Diferenciação Emocional Neutra & 20,14 & 27,40 & 227,00 & 0,279 \\
& Diferenciação Emocional Total & 21,82 & 25,92 & 190,00 & 0,065 \\
& Índice Identificação Emocional & 18,64 & 28,72 & 157,00 & 0,011 \\
\cline { 2 - 3 } Depois & Diferenciação Emocional Positiva & 21,43 & 26,26 & 218,00 & 0,161 \\
& Diferenciação Emocional Negativa & 21,66 & 26,06 & 223,50 & 0,221 \\
& Diferenciação Emocional Neutra & 19,70 & 27,78 & 218,00 & 0,206 \\
& Diferenciação Emocional Total & 21,41 & 26,28 & 180,50 & 0,041 \\
& Índice Identificação Emocional & 19,27 & 28,16 & 171,00 & 0,024 \\
\hline
\end{tabular}

No grupo de adolescentes as ordenações médias são no geral mais elevadas que nas crianças mais novas e igual forma no sexo feminino verificaram-se diferenças estatísticas na diferenciação de emoções positivas e na capacidade de identificar emoçóes, quer antes, $(\mathrm{UMW}=225.500 ; \mathrm{p}=0,032$ e UMW= 189.500: $\mathrm{p}=0,007)$ quer após o programa (UMW= 222.000; $\mathrm{p}=0,024$ e UMW= 191.500; $\mathrm{p}=0,008)$ (Tabela 2).

Tabela 2. Teste de U-Mann Whitney antes e depois do programa de intervenção no grupo de adolescentes, em função do sexo

\begin{tabular}{llcccc}
\hline & & Masc. & Femin. & \multicolumn{2}{c}{ Teste } \\
& & \multicolumn{2}{c}{$12-15$} & UMW & $\boldsymbol{p}$-value \\
\hline \multirow{2}{*}{ Antes } & Diferenciação Emocional Positiva & 21,78 & 30,87 & 225,00 & 0,032 \\
& Diferenciação Emocional Negativa & 25,33 & 28,78 & 296,50 & 0,406 \\
& Diferenciação Emocional Neutra & 22,60 & 30,49 & 238,50 & 0,064 \\
& Diferenciação Emocional Total & 22,43 & 30,38 & 242,00 & 0,076 \\
& Índice Identificação Emocional & 19,98 & 31,93 & 189,50 & 0,007 \\
Depois & Diferenciação Emocional Positiva & 21,60 & 30,97 & 222,00 & 0,024 \\
& Diferenciação Emocional Negativa & 20,30 & 29,80 & 223,50 & 0,221 \\
& Diferenciação Emocional Neutra & 25,03 & 28,96 & 290,50 & 0,363 \\
& Diferenciação Emocional Total & 26,50 & 30,23 & 225,00 & 0,067 \\
& Índice Identificação Emocional & 20,08 & 31,87 & 191,50 & 0,008 \\
\hline
\end{tabular}

Para análise dos resultados em função da idade, foi efetuado o teste não-paramétrico KruskalWallis, dado o tamanho da amostra, evidenciando no grupo global ordenaçóes médias mais elevadas nos alunos de 14 anos, tanto na avaliação antes, como depois do programa. Por grupos e analisando as crianças mais novas, quer antes, quer após o 
Tabela 3. Test-t para diferença de médias antes e depois do programa de intervenção, em função dos grupos etários

\begin{tabular}{|c|c|c|c|c|c|c|c|c|}
\hline \multirow{3}{*}{$\begin{array}{l}\text { Grupos etários } \\
\text { Itens }\end{array}$} & \multirow{3}{*}{$\begin{array}{l}\text { Antes } \\
\text { M(Dp) }\end{array}$} & \multirow{3}{*}{$\begin{array}{l}\text { Depois } \\
\text { M(Dp) }\end{array}$} & \multicolumn{2}{|c|}{ Teste-t } & \multirow{3}{*}{$\begin{array}{l}\text { Antes } \\
\text { M(Dp) }\end{array}$} & \multirow{3}{*}{$\begin{array}{l}\text { Depois } \\
M(D p)\end{array}$} & \multicolumn{2}{|c|}{ Teste-t } \\
\hline & & & \multirow{2}{*}{$\mathrm{t}$} & \multirow{2}{*}{$\mathrm{p}$-value } & & & \multirow{2}{*}{$\mathrm{t}$} & \multirow{2}{*}{$\mathrm{p}$-value } \\
\hline & & & & & & & & \\
\hline Diferenciação Emocional Positiva & $4,29(0,99)$ & $4,72(1,49)$ & $-2,813$ & 0,007 & $5,83(2,45)$ & $5,33(1,91)$ & 1,540 & 0,129 \\
\hline Diferenciação Emocional Negativa & $3,91(0,80)$ & $3,80(1,03)$ & 0,726 & 0,472 & $4,70(1,63)$ & $4,88(1,79)$ & $-0,843$ & 0,403 \\
\hline Diferenciação Emocional Neutra & $3,87(2,39)$ & $3,21(2,55)$ & 2,258 & 0,029 & $3,94(1,99)$ & $4,12(2,14)$ & 0,849 & 0,400 \\
\hline Diferenciação Emocional Total & $8,21(1,45)$ & $8,53(2,14)$ & $-1,342$ & 0,186 & $10,53(1,45)$ & $10,22(3,30)$ & 0,678 & 0,501 \\
\hline Índice Identificação Emocional & $12,08(2,93)$ & $11,74(3,51)$ & 0,884 & 0,31 & $14,48(4,40)$ & $14,35(4,26)$ & 0,232 & 0,817 \\
\hline
\end{tabular}

programa de educação emocional, verificou-se ordenaçôes médias mais elevadas na diferenciação de emoçóes positivas, negativas e diferenciação total nos alunos de 11 anos e nas emoçóes neutras e capacidade de identificação de emoçôes nas de 10 anos, contudo sem significância estatística $(p>0,05)$. Nos adolescentes a diferenciação e identificação emocional foi mais elevada nas crianças de 14 anos quer antes quer após o programa, mas apenas significativa antes do programa na diferenciação de emoções neutras (UMW=9,440; $\mathrm{p}=0,024)$ e identificação de emoções (UMW=10,500; $\mathrm{p}=0,015$ ).

No que se refere à relação com o ano de escolaridade no grupo dos 9-11 anos e utilizando o teste de Kruskal-Wallis, apurou-se, tanto antes como depois do programa, que as ordenaçóes médias eram mais elevadas nos alunos do $5^{\circ}$ ano em todas as dimensôes, exceto na diferenciação emocional negativa e total (avaliação antes) e na diferenciação emocional negativa após o programa, contudo sem significância estatística $(p>0,05)$. No grupo de adolescentes verificou-se ordenaçôes médias mais elevadas nos alunos do $8^{\circ}$ ano na avaliação antes do programa e no $9^{\circ}$ ano após a intervenção educacional, igualmente sem diferenças estatísticas significativas.

A análise dos valores médios da escala antes do programa de educação socio emocional em função dos grupos, indicou que as crianças e adolescentes fazem no geral pouca diferenciação de emoçóes, contudo os valores eram mais elevados em todas as dimensôes no grupo etário dos adolescentes, verificando-se o valor máximo na escala de diferenciação emocional positiva e mínimo na diferenciação emocional neutra, e um índice de identificação emocional fraco (média $=14,48)$, face ao score máximo possível (39), o que indica, apesar dos melhores resultados deste grupo, uma baixa capacidade de diferenciação e identificação emocional. Após o programa de educaçáo socio emocional os valores médios aumentaram apenas na diferenciação emocional negativa e neutra. No grupo etário dos 9-11 anos verificou-se uma tendência idêntica antes, ou seja, valores médios baixos, face aos scores possíveis e após o programa apenas se registou aumento dos valores médios na diferenciação emocional positiva e na escala total (Tabela 1 ).

De forma a avaliarmos o resultado da implementação do programa de educação socio emocional foi utilizado o Test-t para diferença de médias em função da amostra global e dos grupos etários. $\mathrm{Na}$ amostra global não se apuraram diferenças estatísticas significativas entre a diferenciação de emoçôes antes e depois da atividade. Por grupos (Tabela 1) apurou-se apenas nas crianças de 9-11 anos diferenças com significância estatística, nomeadamente na Diferenciação Emocional Positiva, com um aumento dos valores médios, o que indica aumento desta competência $(t=-2,813 ; \mathrm{p}=0,007)$ e na Diferenciação Emocional Neutra, onde se registou o contrário, ou seja, uma diminuição dos valores médios, indicando uma maior dificuldade nesta capacidade, após o programa de educação $(t=2,258$; $\mathrm{p}=0,029)$ (Tabela 3).

\section{Discussão}

Os resultados obtidos no presente estudo, salientam a maior capacidade de diferenciar e identificar emoçôes nas crianças mais velhas, do sexo feminino e reforçam a ideia do impacto da educação neste âmbito, sobretudo nas crianças mais novas.

Apesar da metodologia distinta, também o estudo de Esturgó sobre inteligência emocional e comportamentos disruptivos, realizado com alunos do ensino básica ${ }^{(10-14)}$ evidenciou relações com o género, verificando-se que os rapazes apresentavam mais comportamentos disruptivos do que as rapa- 
rigas e uma relação negativa e significativa entre os comportamentos disruptivos e os índices gerais de inteligência emocional, enquanto as raparigas apresentavam maiores competências socioemocionais e mais comportamentos positivos. ${ }^{(15)}$

$\mathrm{Na}$ literatura científica divulga programas de intervenção socio-emocional em contexto escolar ${ }^{(9-13)}$ que englobam alunos, pais/ encarregados de educação e professores, desenvolvidos com o intuito de alterar práticas, atitudes e crenças e que mostram evidências de uma melhoria significativa nas competências socioemocionais e no sucesso escolar. Esta relação positiva é suportada em investigaçôes sobre Inteligência Emocional $^{(3)}$ que têm demonstrado que pessoas que investem na aprendizagem de competências sociais e emocionais, apresentam uma melhor saúde física e psicológica, melhores relações interpessoais, mais estados emocionais positivos e, portanto, uma atitude mais positiva em relação ao mundo que as rodeia, incluindo a escola e os professores.

Outras investigaçóes realizadas com o mesmo objetivo $^{(16-19)}$ em amostras de alunos do $3^{\circ}$ ciclo, pais e professores apuraram resultados semelhantes aos encontrados no presente estudo, nomeadamente uma maior capacidade de identificar e diferenciar emoçóes nas crianças mais velhas e do sexo feminino. Semelhantes pesquisas salientam ainda como decisiva a formação dos professores envolvidos nos programas de intervenção, condição determinante para que os alunos se envolvam e para uma implementação mais efectiva, estando esta relacionada com resultados mais duradouros nos alunos. ${ }^{(20)}$ Estas intervenções efetuadas sobretudo junto de crianças do ensino básico, no sentido de as capacitar para o reconhecimento e gestão das emoçôes, origina maiores capacidades de, não só, reconhecer mais facilmente os estados emocionais deles e dos outros, como também de os gerir adequando de forma mais acurada as suas interaçóes.

Nos estudos analisados no âmbito desta investigação, ${ }^{(9-19)}$ as conclusões apontam para benefícios como, maior bem-estar, melhores competências relacionais, atitudes mais positivas perante as situações de vida, menos manifestaçôes de comportamentos internalizantes e externalizantes, maior capacidade na utilização de estratégias de "coping", comportamentos mais adaptativos em situaçóes de maior pressão e maior sucesso académico na crianças e jovens envolvidos. ${ }^{(21)}$

No presente estudo os resultados indicam que a aplicação de um programa de identificação de emoçóes a crianças e jovens permitiu melhorar as competências de identificação e diferenciação de algumas emoções, porém, mais dificuldade na diferenciação de outras, o que poderá estar relacionado com a natureza fortuita do programa de intervenção realizado, reforçando a pertinência de uma implementação mais efetiva, se se pretende melhorias significativas e potencialmente duradouras nos alunos.

\section{Conclusão}

A amostra do presente estudo foi constituída por 101 crianças e jovens, sendo a maioria do sexo feminino e dividida nos grupos etários de 9-11 e 1215 anos. O programa de educação sócio emocional foi em parte eficaz na identificação e diferenciação de emoçóes, dado que após a sua implementação foram evidentes melhores competências nas crianças de 9-11 anos, nomeadamente na Diferenciação Emocional Positiva, com um aumento dos valores médios, o que indica aumento desta competência e na Diferenciação Emocional Neutra, onde se registou o contrário, ou seja, uma maior dificuldade nesta capacidade. $\mathrm{O}$ estudo revela algumas limitações metodológicas, associadas às características transversais da sua aplicação, reduzido número de participantes e escasso tempo que mediou entre o estudo antes e após. A falta de um grupo de controlo condiciona de alguma forma a determinação da influência da intervenção na eficácia do programa.

\section{Agradecimentos}

Os nossos agradecimentos ao Fundo Social Europeu, ao Programa Operacional CENTRO 2020 e à Fundação para a Ciência e Tecnologia do Governo Português, instituiçóes públicas que se constituem como fontes de financiamento do Projeto de Investigação MAISaúde Mental "Monitorização 
$e$ avaliação dos indicadores de saúde mental das crianças e adolescentes: Da investigação à prática" (código de identificação:CENTRO-01-0145-FEDER-023293), onde este estudo se integra.

\section{Colaborações}

Campos SMG, Costa MGA, Ferreira M, Ribeiro OP, Costa S, Duarte J, Martins R e Albuquerque C contribuíram com a conepção do projeto, análise e interpretação dos dados, redação do artigo, revisão crítica relevante do conteúdo intelectual e aprovação da versão final a ser publicada.

\section{Referências}

1. Damasio A. Ao encontro de Espinoza. Lisboa: Edicao Temas e Debates; 2012.

2. Grossmann K, Grossmann KE. 0 impacto do apego a mãe e ao pai e do apoio sensível a exploração nos primeiros anos de vida sobre 0 desenvolvimento psicossocial das crianças ate 0 inicio da vida adulta. In: Tremblay RE, Boivin M, Peters RD, editors. Enciclopedia sobre 0 desenvolvimento na primeira infância [Internet]. Centro de Excelencia para o Desenvolvimento na Primeira Infância; 2011. [citado 2019 Jan 8]. Disponivel em: http://www.enciclopedia-crianca.com/sites/default/ files/dossiers-complets/pt-pt/apego.pdf

3. Caruso DR, Mayer JD, Salovey P. Relation of an ability measure of emotional intelligence to personality. J Pers Assess. 2002;79(2):306-20.

4. ins JE, Elias MJ. Social and Emotional Learning: promoting the development of all students. J Educ Psychol Consult. 2007;17(2-3):233-55.

5. Portugal. Ministerio da Saude. Direcao-Geral da Saude. Programa Nacional de Saude Escolar. [Internet]. 2015 [citado 2019 Jan 14]. Disponível em: https://observatoriolisboa.eapn.pt/.../ProgramaNacional-de-Saude-Escolar-2015.pdf

6. Moreira P, Oliveira JT, Crusellas L, Lima A. Inventario de Identificação de Emoções e Sentimentos (IIES): Estudo de desenvolvimento e de validação. Rev Psicol Criança Adolesc. 2012;3(1):39-66.

7. Mayer JD, Salovey P. What is emotional intelligence? In: Salovey P, Sluyter DJ, editors. Emotional development and emotional intelligence: Implications for educator. New York: Basic Books; 1997. p. 3-31.

8. Ekman P. El rosto de las emociones: Signos que revelan significado mas alla de las palabras. Barcelona: RBA Libros; 2012.

9. Payton JW, Wardlaw DM, Graczyk PA, Bloodworth MR, Tompsett CJ, Weissberg RP. Social and emotional learning: a framework for promoting mental health and reducing risk behavior in children and youth. J Sch Health. 2000;70(5):179-85.
10. Greenberg MT, Weissberg RP, O'Brien MU, Zins JE, Fredericks L, Resnik $\mathrm{H}$, et al. Enhancing school-based prevention and youth development through coordinated social, emotional, and academic learning. Am Psychol. 2003;58(6-7):466-74.

11. Catalano RF, Berglund ML, Ryan JA, Lonczak HS, Hawkins JD. Positive youth development in the United States: Research findings on evaluations of positive youth development programs. Prevent Treat. 2002; 5(1). Article ID 15.

12. Durlak JA, Weissberg RP, Dymnicki AB, Taylor RD, Schellinger KB. The impact of enhancing students' social and emotional learning: a meta-analysis of school-based universal interventions. Child Dev. 2011;82(1):405-32.

13. Webster-Stratton C, Reid MJ, Beauchaine TP. One-year follow-up of combined parent and child intervention for young children with ADHD. J Clin Child Adolesc Psychol. 2013;42(2):251-61.

14. Esturgo-Deu ME, Sala-Roca J. Disruptive behaviour of students in primary education and emotional intelligence. Teach Teach Educ. 2010;26(4):830-7.

15. Trentacosta CJ, Mostow AJ, Izard CE, Fine SE. Children's emotional competence and attentional competence in early elementary school. Sch Psychol Q. 2006;21(2):148-70.

16. Ferreira M, Duarte J, Campos S, Chaves C, Felizardo S, Cardoso AP. Emotional skills and promotion of school success: Preliminary data of an intervention programme with parents. In: The European Proceedings of Social \& Behavioural Science. 3rd International Conference on Health and Health Psychology; 2017 Jul 5-7; Porto, Portugal [citado 2019 Jan 12]. London: Future Academy; 2017. p. 310-7. Disponivel em: http://dx.doi.org/

17. Cardoso AP, Ferreira M, Campos S, Duarte J, Chaves C, Felizardo S. Emotional competences and promotion of school success: Preliminary data of an intervention programme with teachers. In: Gomez Chova L, Lopez Martinez A, Candel Torres I, editors. Proceedings of ICERI2016 Conference; 2016 Nov 14-16 th; Seville. Seville (Spain): IATED Academy; 2016. p. 922-7. https:// doi.org/10.21125/iceri.2016.1209.

18. Campos S, Ferreira M, Cardoso AP, Duarte J, Felizardo S, Chaves C. Emotional skills and promoting school success in the 3rd cycle: Students perception. In: The European Proceedings of Social \& Behavioural Science. 7th International Conference on Education and Educational Psychology; 2016 Out 11-15; Rhodes, Greece [citado 2019 Jan 18]. London: Future Academy; 2016. p. 502-11. Disponivel em: http://dx.doi.org/

19. Duarte J, Ferreira M, Chaves C, Campos S, Felizardo S, Cardoso AP, et al. Emotional intelligence: The self-evaluation of emotional behavior, problems and skills in students of the 3rd cycle of basic education. In: Vranesevic T, Seabra C, Perucic D, Mandic M, Pandza I, editores. 6th International M-Sphere Conference for Multidisciplinarity in Business and Science: Book of abstracts; 2017 Sep 19-22; Viseu, Portugal. Zabreg, Croatia: M-Sphere; 2017. p.76-7.

20. Greenberg MT, Domitrovich CE, Weissberg RR, Durlak JA. Social and emotional learning as a public health approach to education. Future Child. 2017; 27(1): 13-32

21. MacCann C, Fogarty GJ, Zeidner M, Roberts RD. Coping mediates the relationship between emotional intelligence (El) and academic achievement. Contemp Educ Psychol. 2011;36(1):60-70. 